\title{
Reducing and meta-analysing estimates from distributed lag non-linear models
}

\author{
Antonio Gasparrini ${ }^{*}$ and Ben Armstrong ${ }^{2}$
}

\begin{abstract}
Background: The two-stage time series design represents a powerful analytical tool in environmental epidemiology. Recently, models for both stages have been extended with the development of distributed lag non-linear models (DLNMs), a methodology for investigating simultaneously non-linear and lagged relationships, and multivariate meta-analysis, a methodology to pool estimates of multi-parameter associations. However, the application of both methods in two-stage analyses is prevented by the high-dimensional definition of DLNMs.

Methods: In this contribution we propose a method to synthesize DLNMs to simpler summaries, expressed by a reduced set of parameters of one-dimensional functions, which are compatible with current multivariate meta-analytical techniques. The methodology and modelling framework are implemented in $\mathrm{R}$ through the packages dlnm and mvmeta.

Results: As an illustrative application, the method is adopted for the two-stage time series analysis of temperature-mortality associations using data from 10 regions in England and Wales. R code and data are available as supplementary online material.

Discussion and Conclusions: The methodology proposed here extends the use of DLNMs in two-stage analyses, obtaining meta-analytical estimates of easily interpretable summaries from complex non-linear and delayed associations. The approach relaxes the assumptions and avoids simplifications required by simpler modelling approaches.
\end{abstract}

Keywords: Distributed lag models, Multivariate meta-analysis, Two-stage analysis, Time series

\section{Background}

Research on the health effects of environmental stressors, such as air pollution and temperature, often relies on time series analysis using data from multiple locations, usually cities [1,2]. The analytical design adopted in this setting is commonly based on two-stage procedures, where location-specific exposure-response relationships are estimated through a regression model in the first stage, and these estimates are then combined through meta-analysis in the second stage [3].

Recently, the first-stage modelling approaches have been extended with the introduction of distributed lag nonlinear models (DLNMs) [4,5], a methodology to describe simultaneously non-linear and delayed dependencies.

\footnotetext{
*Correspondence: antonio.gasparrini@lshtm.co.uk

${ }^{1}$ Department of Medical Statistics, London School of Hygiene and Tropical

Medicine, London, UK

Full list of author information is available at the end of the article
}

This modelling class is based on the definition of a crossbasis, a bi-dimensional space of functions describing the association along the spaces of predictor and lags. The cross-basis is specified by the choice of two basis, one for each dimension, among a set of possible options such as splines, polynomials, or step functions. Concurrently, developments have been proposed also for the second stage. In particular, techniques based on multivariate meta-analysis have been used to combine estimates of associations defined by multiple parameters, and applied for either non-linear [6-8] or lagged dependencies [8-11]. We recently provided an overview of the use of multivariate meta-analysis in this setting [12].

In this contribution we propose a method to reduce estimates from DLNMs to summaries defined in only one dimension of predictor or lags, re-expressing the fit in terms of reduced parameters for the related uni-dimensional basis functions. This step decreases the 
number of parameters to be pooled in the second stage, offering a method to meta-analyse estimates from richly parameterized non-linear and delayed exposure-response relationships.

In the next section, we provide a brief recap of the algebraic development of DLNMs and multivariate metaanalysis, and then describe the main statistical development, establishing a method to reduce the fit of a DLNM to summaries expressed in a single dimension. A motivating example with an analysis of the relationship between temperature and all-cause mortality is used throughout the paper to illustrate the statistical framework. We finally note some limitations and indicate future directions for research. Supplementary online material provides information on algebraic notation and software (Additional file 1), and also includes the $\mathrm{R}$ code and data to entirely reproduce the results in the example (Additional files 2-3).

\section{Methods}

The two-stage time series design can be applied to series of observations collected at each time $t$, with $t=1, \ldots, N_{i}$, in each location $i$, with $i=1, \ldots, m$. First-stage regression models are fitted to each series of $N_{i}$ observations, obtaining location-specific estimates of the association of interest. These estimates are then pooled across locations in the second stage, with the aim to estimate an average exposure-response relationship and inspect heterogeneity across locations.

\section{An illustrative example}

As an illustration, we describe an analysis of the relationship between temperature and all-cause mortality using daily series of $N_{i}=N=5113$ observations from each of the $m=10$ regions in England and Wales, in the period 1993-2006. Further details on the dataset were previously provided $[13,14]$. The example is used throughout the paper to describe the steps of the modelling framework and introduce the new methodological development. Specifically, the relationship is flexibly estimated in each region in the first-stage analysis using DLNMs, and then pooled in the second stage through multivariate meta-analysis. The example aims to demonstrate how results from DLNMs are summarized in an analysis of a single region, and then how these reduced summaries can be combined across regions. Also, we illustrate a comparison with simpler modelling approaches. Modelling choices are dictated by illustrative purposes, and the results are not meant to provide substantive evidence on the association.

\section{Distributed lag non-linear models}

The DLNM framework has been extensively described [5]. Here we provide a brief overview to facilitate the new development, illustrated later. In particular, we will focus on the bi-dimensional structure of this class of models, represented by the two sets of basis functions applied to derive the parameterization. Following the original paper, we first generalize the idea of simple distributed lag models (DLMs) and then introduce the non-linear extension.

\section{The DLNM modelling class}

Distributed lag linear and non-linear models are expressed through a lag-basis and cross-basis function $s\left(x_{t}\right)$, respectively, of the $N$-length exposure series $\mathbf{x}=\left[x_{1}, \ldots, x_{t}, \ldots, x_{N}\right]^{\top}$. The definition of $s\left(x_{t}\right)$ first requires the derivation of the $N \times(L+1)$ matrix $\mathbf{Q}$ of lagged exposures, so that $\mathbf{q}_{t .}=\left[x_{t}, \ldots, x_{t-\ell}, \ldots, x_{t-L}\right]^{\top}$. This step indirectly characterizes the new lag dimension identified by the vector $\ell=[0, \ldots, \ell, \ldots, L]^{\top}$, with $L$ as maximum lag. Now, choosing a first basis with dimension $\nu_{\ell}$ to represent the association along the new lag space, we can compute a $(L+1) \times v_{\ell}$ basis matrix $\mathbf{C}$ by applying the related functions to $\ell$. A compact and general definition of the lag-basis function $s\left(x_{t}\right)$ for DLMs is given by:

$$
s\left(x_{t} ; \boldsymbol{\eta}\right)=\sum_{k=1}^{v_{\ell}} \mathbf{q}_{t}^{\top} \cdot \mathbf{c}_{\cdot k} \eta_{k}=\mathbf{q}_{t}^{\top} \cdot \mathbf{C} \boldsymbol{\eta}=\mathbf{w}_{t}^{\top} \cdot \boldsymbol{\eta},
$$

where different models are specified with different choices of the basis to derive $\mathrm{C}$. The transformed variables in $\mathbf{W}=\mathbf{Q C}$ can be included in the design matrix of the firststage regression model, in order to estimate the $v_{\ell}$-length parameter vector $\boldsymbol{\eta}$, with $\mathbf{C} \boldsymbol{\eta}$ representing the lag-specific contributions.

The non-linear extension to DLNMs requires the choice of a second basis with dimension $v_{x}$ to model the relationship along the space of the predictor $x$, obtaining the $N \times v_{x}$ basis matrix $\mathbf{Z}$ from the application of the related functions to $\mathbf{x}$. Applied together with the transformation which defines the matrix of lagged exposures $\mathbf{Q}$ above, this step produces a three-dimensional $N \times v_{x} \times(L+1)$ array $\dot{\mathbf{R}}$. The parameterization of the cross-basis function $s\left(x_{t}\right)$ for DLNMs is then given by:

$$
s\left(x_{t} ; \boldsymbol{\eta}\right)=\sum_{j=1}^{v_{x}} \sum_{k=1}^{v_{\ell}} \mathbf{r}_{t j}^{\top} \cdot \mathbf{c}_{\cdot k} \eta_{j k}=\mathbf{w}_{t}^{\top} \cdot \boldsymbol{\eta} .
$$

The simpler lag-basis for DLMs in (1) is a special case of the more complex cross-basis for DLNMs in (2). These models may be fitted through common regression techniques with the inclusion of cross-basis matrix $\mathbf{W}$ in the design matrix. The vector $\hat{\eta}$ of estimated parameters of the cross-basis function in (2) represents a simultaneously non-linear and lagged dependency, and its length $v_{x} \times v_{\ell}$ is equal to the product of the dimensions of the bases for the two spaces. In completely parametric models as those described here, this dimensionality is directly associated with the notion of degrees of freedom $(d f)$, related 
to the flexibility of the function and the smoothness of the estimated dependency. In spite of the relatively complex algebraic definition in (2), DLNMs are solely specified by the choice of the two bases for deriving the matrices $\mathbf{Z}$ and C. The software implementation of this methodology in the $R$ package dlnm has been previously described [15].

\section{Summarizing the results from a DLNM}

Fitted bi-dimensional cross-basis functions from DLNMs can be interpreted by deriving predictions over a grid of predictor and lag values, usually computed relative to a reference predictor value. As a first example, we show the results of a single-location analysis, using data from the North-East region of England. The temperature-mortality relationship is modelled through the same cross-basis used for the full two-stage analysis illustrated later, composed by two B-spline bases.

The results are shown in Figure 1. The top-left panel displays the bi-dimensional surface of the fitted relative risk (RR) in a 3-D graph, predicted for the grid of temperature and lag values, with a reference black line corresponding to the centering value of the basis for the predictor space (here $17^{\circ} \mathrm{C}$ ). Similarly to previous analyses, the figure suggests an immediate increase in risk for high temperature, and a more delayed but protracted effect for low temperature.

This bi-dimensional representation contains details not relevant for some interpretative purposes, and does not easily allow presentation of confidence intervals. The analysis therefore commonly focuses on three specific uni-dimensional summaries of the association, also illustrated in Figure 1. First, a predictor-specific summary association at a given predictor value $x_{0}$ can be defined along the lag space. As an example, this is reproduced in the top-right panel for temperature $x_{0}=22^{\circ} \mathrm{C}$, together with $95 \%$ confidence intervals $(\mathrm{CI})$, and corresponds to the red line parallel to the reference in the 3-D graph. Second, similarly, a lag-specific summary association at a given lag value $\ell_{0}$ can be defined along the predictor space. This is shown in the bottom-left panel for lag $\ell_{0}=4$, and coincides with the red line in the 3-D graph perpendicular to the reference. Third, the sum of the lag-specific contributions provides the overall cumulative association, showed in the bottom-right panel of Figure 1. This last summary offers an estimate of the net effect associated with a given exposure cumulated over the lag period $L$, and is usually the focus of the analysis.

\section{Multivariate meta-analysis}

The framework of multivariate meta-analysis has been previously described [16], and its application for combining estimates of multi-parameter associations has been recently illustrated [12]. We offer a brief summary here, firstly illustrating the second-stage multivariate meta-analytical model and then discussing its limitation for pooling DLNMs.

\section{The multivariate extension of meta-analysis}

Specification of the model assumes that a $k$-dimensional set of outcome parameters $\hat{\boldsymbol{\eta}}_{i}$ and associated $k \times k$ (co)variance matrix $\mathbf{S}_{i}$ have been estimated in each of the $i=1, \ldots, m$ studies. In the application for two-stage time series analysis, these outcome parameters represent regression coefficients from the first stage, while the term study refers here to the first-stage analysis in each location. The description below illustrates a random-effects multivariate meta-regression model, where fixed-effects models or simple meta-analysis treated as special cases. The model for location $i$ is defined as:

$$
\hat{\boldsymbol{\eta}}_{i} \sim \mathrm{N}\left(\mathbf{U}_{i} \boldsymbol{\beta}, \mathbf{S}_{i}+\boldsymbol{\Psi}\right)
$$

where the location-specific estimated outcome parameters $\hat{\boldsymbol{\eta}}_{i}$ are assumed to follow a $k$-dimensional multivariate normal distribution. The $k \times k p$ block-diagonal matrix $\mathbf{U}_{i}=\mathbf{I}_{(k)} \otimes \mathbf{u}_{i}^{\top}$ is the Kronecker expansion of the locationspecific vector $\mathbf{u}_{i}=\left[u_{1}, \ldots, u_{p}\right]^{\top}$ of meta-variables. The matrices $\boldsymbol{\Psi}$ and $\mathbf{S}_{i}$ represent the between and withinlocation (co)variance matrices, respectively. This multivariate meta-regression model is applied to estimate the parameter vectors $\boldsymbol{\beta}$ and $\boldsymbol{\xi}$. The former represents the $k p$ second-stage coefficients defining how the $p$ metavariables are associated with each of the true $k$ first-stage coefficients in $\boldsymbol{\eta}_{i}$. The vector $\boldsymbol{\xi}$ includes a set of parameters which uniquely define the between-location (co)variance matrix $\Psi$, depending on the chosen structure for this matrix. In fixed effects models no additional variability beyond the estimation error in the first-stage model is assumed for $\hat{\boldsymbol{\eta}}_{i}$, and $\boldsymbol{\Sigma}_{i}=\mathbf{S}_{i}$. In multivariate metaanalysis with no meta-variable, $\mathbf{U}=\mathbf{I}_{(k)}$ and $\boldsymbol{\beta}=\boldsymbol{\eta}$, the vector of average parameters. The development in (3) can be considered as a special case of multivariate linear mixed model [17], where the within-city (co)variance is assumed known. Among alternative estimation methods, such as Bayesian [18] and multivariate extensions of the method of moments [19], we privilege here likelihoodbased approaches [20,21]. Methods to derive tests and confidence intervals, fit statistics and (best-linear unbiased) predictions have been previously developed within the linear mixed models framework for the application in this setting, together with a description of the software implementation in the $\mathrm{R}$ package mvmeta [12].

\section{Limitations of multivariate meta-analysis}

In theory, the $m$ sets of estimated first-stage coefficients $\hat{\boldsymbol{\eta}}_{i}$ of the cross-basis obtained from DLNMs 


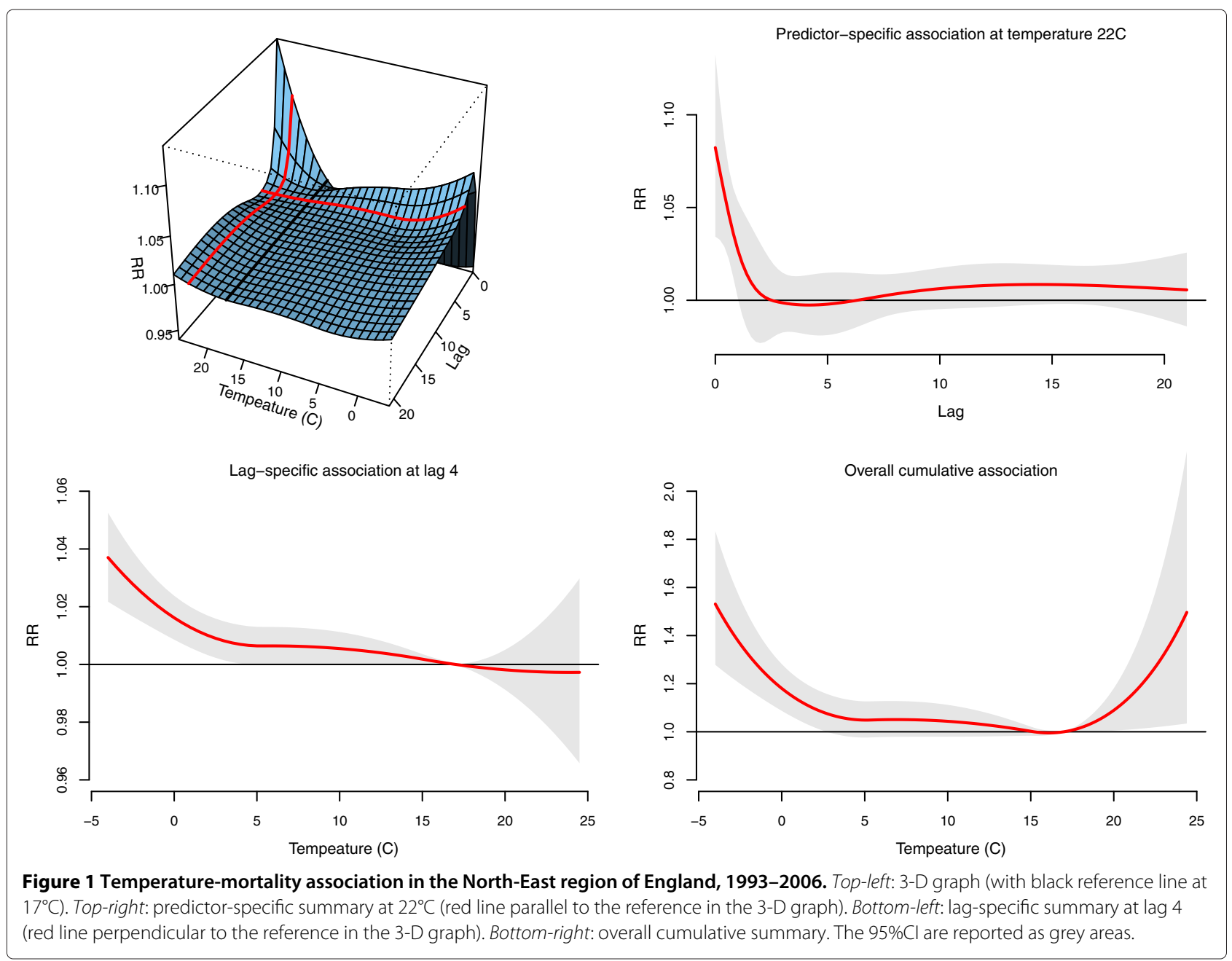

in (2) can be meta-analysed using (3), producing an population-averaged three-dimensional effect surface across locations, optionally conditional on meta-variables in multivariate meta-regression. However, as anticipated above, the definition of DLNMs in (2) requires $k=v_{x} \times$ $v_{\ell}$ parameters $\hat{\boldsymbol{\eta}}_{i}$ for the cross-basis. For models specified by even moderately complex bases in each space, the number of parameters becomes so high that the optimization routine for multivariate meta-analysis is computationally impractical. This is particularly relevant for the (co)variance terms in $\xi$ defining the true between-location variability, composed by $k(k+1) / 2$ parameters for an unstructured matrix $\Psi$.

This limitation is one of the main reasons which have prevented so far the full application of DLNMs in twostage analysis. The modelling approach has often required the simplification of the first-stage model, for the secondstage multivariate meta-analysis to be feasible. For example, investigators have assumed a linear relationship in the dimension of the predictor [8-11], or computed a simple exposure moving average for the lag space [6-8]. We previously adopted the same limited approach [12]. The development of methods to derive meta-analytical estimates from full DLNMs would offer a great deal of flexibility in the investigation of complex exposure-response dependencies.

\section{Reducing DLNMs}

Predictions from DLNMs as those shown in Figure 1 are obtained by selecting the grid of exposure and lag values defined as $\mathbf{x}_{[p]}$ and $\boldsymbol{\ell}_{[p]}$, respectively. Details on the algebraic development are given elsewhere [5] [sections 4.2-4.3]. Briefly, predictions are computed by deriving matrices $\mathbf{Z}_{[p]}$ and $\mathbf{C}_{[p]}$ from $\mathbf{x}_{[p]}$ and $\boldsymbol{\ell}_{[p]}$, respectively, through the application of the same basis functions used for estimation in (2). The bi-dimensional predicted relationship in location $i$ is expressed by the full set of estimated parameters in $\hat{\boldsymbol{\eta}}_{i}$ and quantities derived by $\mathbf{Z}_{[p]}$ and $\mathbf{C}_{[p]}$. However, the specific summaries described in the previous section are defined only on the single dimension of predictor (lag-specific and overall cumulative associations, bottom panels of Figure 1) or lag (predictor-specific 
association, top-right panel of Figure 1). The idea is to re-parameterize these summaries in terms of the related uni-dimensional basis $\mathbf{Z}_{[p]}$ or $\mathbf{C}_{[p]}$ for predictor or lags only, respectively, and sets of reduced in coefficients $\hat{\boldsymbol{\theta}}_{i}$. Dimensionality of the functions expressing such summaries therefore decreases from $v_{x} \times v_{\ell}$, corresponding to the length of vector $\hat{\eta}_{i}$ in the original parameterization, to $v_{x}$ or $v_{\ell}$ only, corresponding to the length of new sets of reduced parameters $\hat{\boldsymbol{\theta}}_{i}$.

The definition of the reduced parameters depends on the specific summary among those listed above. They can be obtained by applying a related dimension-reducing matrix $\mathbf{M}$, expressed as:

$$
\begin{aligned}
& \mathbf{M}_{\left[x_{0}\right]}=\mathbf{I}_{\left(v_{\ell}\right)} \otimes \mathbf{z}_{\left[x_{0}\right]}^{\top} \\
& \mathbf{M}_{\left[\ell_{0}\right]}=\mathbf{c}_{\left[\ell_{0}\right]}^{\top} \otimes \mathbf{I}_{\left(v_{x}\right)} \\
& \mathbf{M}_{[c]}=\mathbf{1}_{(L+1)}^{\top} \mathbf{C} \otimes \mathbf{I}_{\left(v_{x}\right)}
\end{aligned}
$$

for predictor-specific summary association at $x_{0}$, for for lag-specific summary association at $\ell_{0}$, and for overall cumulative summary association, respectively. Here $\mathbf{z}_{\left[x_{0}\right]}$ and $\mathbf{c}_{\left[\ell_{0}\right]}$ are the vectors of transformed values of $x_{0}$ and $\ell_{0}$ obtained by the application of the sets of basis functions for predictor and lags, respectively. The reduced parameter vector $\hat{\boldsymbol{\theta}}_{i}$ and associated (co)variance matrix $V\left(\hat{\boldsymbol{\theta}}_{i}\right)$ are then obtained by:

$$
\begin{gathered}
\hat{\boldsymbol{\theta}}_{[j] i}=\mathbf{M}_{[j]} \hat{\boldsymbol{\eta}}_{i} \\
V\left(\hat{\boldsymbol{\theta}}_{[j] i}\right)=\mathbf{M}_{[j]} V\left(\hat{\boldsymbol{\eta}}_{i}\right) \mathbf{M}_{[j]}^{\top},
\end{gathered}
$$

with $j \in\left\{x_{0}, \ell_{0}, c\right\}$. The predictor-specific association at $x_{0}$, defined on the lag space for values in $\ell_{[p]}$, is expressed by $\mathbf{C}_{[p]} \hat{\boldsymbol{\theta}}_{\left[x_{0}\right] i}$, with standard errors provided by the square root of $\mathbf{C}_{[p]} V\left(\hat{\boldsymbol{\theta}}_{\left[x_{0}\right] i}\right) \mathbf{C}_{[p]}^{\top}$. The lag-specific association at $\ell_{0}$ and the overall cumulative association, defined on the predictor dimension for values in $\mathbf{x}_{[p] i}$, are then obtained by $\mathbf{Z}_{[p]} \hat{\boldsymbol{\theta}}_{\left[\ell_{0}\right] i}$ and $\mathbf{Z}_{[p]} \hat{\boldsymbol{\theta}}_{[c] i}$, respectively, with computation for standard errors as above. The number of coefficients defining these summaries, reduced from $v_{x} \times v_{\ell}$ to $v_{\ell}$ or $v_{x}$ only, is usually compatible with the application of standard multivariate meta-analysis techniques, which can now be used to combine the estimates of these summaries from DLNMs in two-stage analyses. In addition, the derivation in (4)-(5) simplifies the algebra for DLNM predictions originally provided [5] [Section 4.3]. The dimension reduction comes at the price of loss of information about the association on one of the two dimensions, as the rankdeficiency of $\mathbf{M}$ does not allow reversing the reduction applied in (5).

\section{Results}

The analysis is now extended to the full set of 10 regions, with the aim to produce pooled estimates of the overall cumulative association, and to compare the results with those obtained by simpler approaches, applying a moving average to the daily exposure series. Also, we investigate the lag structure for exposure to cold and hot temperatures through predictor-specific estimates. Finally, we assess heterogeneity and then the role of meta-variables through multivariate meta-regression.

\section{Modelling strategy}

The first-stage region-specific model is specified by adopting a standard analytical approach for time series environmental data $[1,3,4]$. In each region, we fit a common generalized linear model for the quasi-Poisson family to the series of all-cause mortality counts. The model includes the cross-basis for daily mean temperature, a natural cubic spline of time with $10 d f$ /year to control for the long-term and seasonal variation, and indicator variables for day of the week.

In the main first-stage model, the temperature-mortality association is estimated by a flexible cross-basis defined by a quadratic B-spline for the space of temperature, centered at $17^{\circ} \mathrm{C}$, and a natural cubic B-spline with intercept for the space of lags, with maximum lag $L=21$. We place two internal knots at equally spaced values along temperature $\left(5.3^{\circ} \mathrm{C}\right.$ and $\left.15.1^{\circ} \mathrm{C}\right)$ and three internal knots at equallyspaced log-values of lag (1.0, 2.8 and 7.6), with boundary knots at $-4.4^{\circ} \mathrm{C}$ and $24.9^{\circ} \mathrm{C}$, and 0 and 21 lags, respectively. These choices define spline bases with dimensions $v_{x}=4$ and $v_{\ell}=5$ for temperature and lag spaces, respectively. The same specification was previously applied for the single-region analysis.

The set of $v_{x} \times v_{\ell}=20$ coefficients of the cross-basis variables with associated (co)variance matrices, estimated in each region, are then reduced. Specifically, for region $i$ we derive the vector $\hat{\boldsymbol{\theta}}_{[c] i}$ with 4 reduced parameters of the quadratic $\mathrm{B}$-spline of temperature $\mathbf{Z}_{[p]}$ for the overall cumulative summary association, and two vectors $\hat{\boldsymbol{\theta}}_{[0] i}$ and $\hat{\boldsymbol{\theta}}_{[22] i}$ with sets of 5 reduced parameters of the natural cubic B-spline of lags $\mathbf{C}_{[p]}$ for predictor-specific summary associations at $0^{\circ} \mathrm{C}$ and $22^{\circ} \mathrm{C}$. These temperatures correspond approximately to the $1^{\text {st }}$ and $99^{\text {th }}$ of the pooled temperature distribution, respectively. These effects along lags are interpreted using the reference of $17^{\circ} \mathrm{C}$.

For comparison with methods not requiring dimensionality reduction, in two alternative first-stage models we simplify the lag structure by fitting one-dimensional splines to the moving average of the temperature series over lag 0-3 and 0-21, respectively. Such moving average models have been commonly used in weather and air pollution epidemiology $[4,10,22]$. These alternatives 
can be described as DLNMs including cross-bases with a constant function to represent the relationship in the lag space, while keeping the same quadratic B-spline for the space of the predictor, as described for the main model above. In these simplified models, the dimension of fitted relationship does not need to be reduced. In fact, the application of the reduction method returns the original $v_{x} \times v_{\ell}=4 \times 1=4$ parameters re-scaled by the number of lags, giving a dimension-reducing matrix $\mathbf{M}_{[c]}$, as described in (4c), composed in this case by a diagonal matrix with entries corresponding to a constant equal to the number of lags.

The coefficients for each of the three summary associations from the main model are estimated in the 10 regions and then independently included as outcomes in three multivariate meta-analytical second-stage models. The ten estimated sets of coefficients from the two alternative models (equivalent to the overall cumulative summary) were directly meta-analysed. All the second-stage models are fitted here through restricted maximum likelihood (REML) using the R package mvmeta. We first derive an estimate of the pooled relationship through multivariate meta-analysis, and then extend the results showing an example of multivariate meta-regression which includes population-averaged regional latitude as a meta-variable. The effect of latitude is displayed by predicting the averaged temperature-mortality associations for the $25^{\text {th }}$ and $75^{\text {th }}$ percentiles of its distribution, using the same baseline reference of $17^{\circ} \mathrm{C}$. The significance of such an effect is assessed through a Wald test, given a likelihood ratio test cannot be applied to compare model fitted with REML and different fixed-effects structures [12].

\section{Two-stage analysis}

The overall temperature-mortality associations in the 10 regions of England and Wales are illustrated in Figure 2. The left panel shows the regions-specific summary associations from the first stage, together with the pooled average from multivariate meta-analysis, as predicted by the main flexible model. Regions-specific estimates show similar curves, although some variability exists, in particular at the extremes. Consistently with previous findings, the pooled curve suggests an increase in relative risk (RR) for both cold and hot temperatures, although less pronounced for the latter, and with a steeper increase for extreme when compared to mild cold. The average point of minimum mortality is at $17.1^{\circ} \mathrm{C}$, corresponding approximately to the $90^{\text {th }}$ percentile of the pooled temperature distribution. The multivariate Cochran $\mathrm{Q}$ test for heterogeneity is highly significant ( $p$-value $<0.001$ ), and the related $I^{2}$ statistic indicates that $63.7 \%$ of the variability is due to true heterogeneity between regions.

The right panel of Figure 2 illustrates the comparison with the two alternative simpler models. We see that the association based on the $0-21$ lag moving average temperature approximates that based on a flexible DLNM in the cold range, but completely misses the heat effect. The reverse is true for the association based on the $0-3$ lag moving average temperature.

Figure 3 depicts the pooled estimate from the main model for predictor-specific summary associations at $22^{\circ} \mathrm{C}$ and $0^{\circ} \mathrm{C}$, with the same reference of $17^{\circ} \mathrm{C}$, as predicted by the two sets of $\nu_{\ell}=5$ reduced coefficients. Consistently with previous research, the effect of hot temperature is immediate and disappears after 1-2 days, while cold temperatures are associated with mortality for a long lag period, after an initial protective effect. This complex lag pattern can explain the different results provided by the less flexible alternative models. The pooled overall RR estimated by the main model, cumulated along lags for these specific summaries and reported graphically in Figure 2 (left panel), are 1.101 (95\%CI: 1.078-1.124) for $22^{\circ} \mathrm{C}$ and and 1.308 (95\% CI: $\left.1.245-1.375\right)$ for $0^{\circ} \mathrm{C}$, respectively. The Cochran $\mathrm{Q}$ test is significant for the lag curve at $0^{\circ} \mathrm{C}(p$-value $<0.001)$, but not for that at $22^{\circ} \mathrm{C}$ ( $p$-value $=$ $0.178)$, with an $I^{2}$ of $63.4 \%$ and $16.0 \%$, respectively.

It is interesting to note that the second-stage multivariate meta-analytical model for the predictor-specific summary associations at $22^{\circ} \mathrm{C}$ estimates perfectly correlated random components, with between-study correlations equal to -1 or 1 . This is a known phenomenon in multivariate meta-analysis, frequently occuring in the presence of a small number of studies and/or a high within-study uncertainty relative to the between-study variation [23]. However, in this case, the results from the Cochran Q test suggest that a fixed-effects multivariate model may be preferable, and as expected, this model returns almost identical estimates for the pooled summary associations (results not shown).

The heterogeneity across regions can be partly explained as effect modification by region-specific variables. The results of the example of meta-regression with latitude are illustrated in Figure 4. The top panel suggests a differential overall cumulative association between northern and southern regions, a pattern previously reported $[8,10]$. Interestingly, the effect modification seems to occur for cold, with a higher effect in southern regions, but not for heat. The estimated pooled RR at $0^{\circ} \mathrm{C}$ versus $17^{\circ} \mathrm{C}$ are 1.380 (95\%CI: $\left.1.337-1.424\right)$ and 1.237 (95\%CI: $1.198-1.277$ ) for the $25^{\text {th }}$ and $75^{\text {th }}$ percentiles of latitude, respectively, while the same estimates are 1.106 (95\%CI: $1.079-1.133)$ and 1.104 (95\%CI: $1.059-1.150)$ for $22^{\circ} \mathrm{C}$. Overall, the evidence for an effect modification is substantial, with a highly significant Wald test ( $p$-value $<0.001)$. Latitude explains much of the heterogeneity across regions, with an $I^{2}$ reduced to $18.7 \%$ and a non-significant Cochran $Q$ test $(p$-value $=0.174)$. The bottom panels illustrates the same effect modification for 


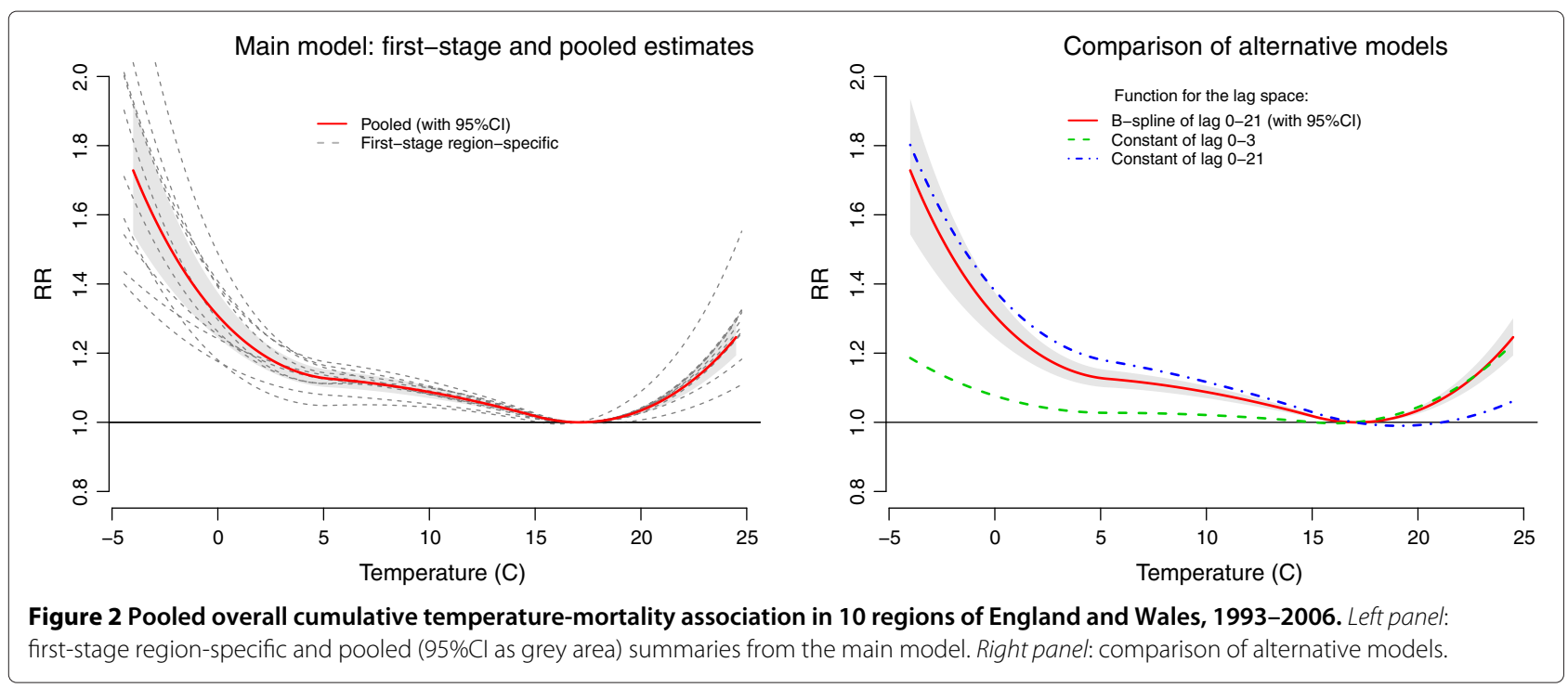

predictor-specific summary associations at $22^{\circ} \mathrm{C}$ and $0^{\circ} \mathrm{C}$. Consistently, the Wald test indicates a significant effect for cold $(p$-value $<0.001)$, but not for heat $(p$-value $=0.634)$.

\section{Discussion}

In this contribution we describe a method to re-express the bi-dimensional fit of DLNMs in terms of unidimensional summaries, involving reduced sets of modified parameters of the basis functions chosen for the space of predictor or lags. This development, in addition to simplifying the algebraic definition of the methodology, offers a more compact description of the bi-dimensional association modelled by DLNMs. In particular, the dimension of the sets of reduced parameters is usually compatible with the application of multivariate meta-analytical techniques in a two-stage framework, allowing the analysis of complex non-linear and delayed associations in multilocation studies.

Previous applications of the two-stage design for multilocation time series studies are based on simplified functions for modelling the association of interest at the first stage. In particular, the analyses are usually limited to splines or other non-linear functions of simple moving average of the exposure series [6-8], a modelling approach similar to the alternative models used for comparison in our example. Alternatively, the simplification could be applied in the other dimension of predictor, specifying DLMs for linear or linear-threshold exposure-response relationships [8-11]. All these approaches require strong assumptions on the exposure-response dependency, in order to simplify the association modelled in one of the two dimensions within the first stage. These are prone
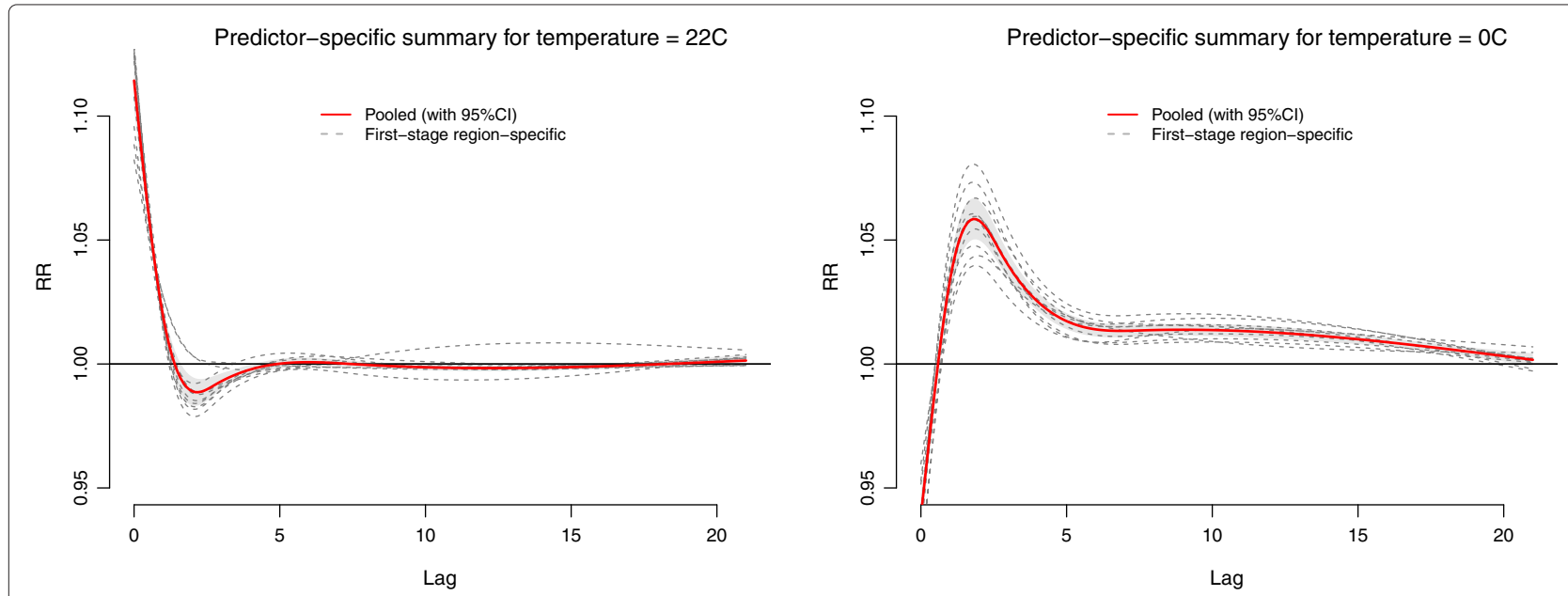

Figure 3 Pooled predictor-specific temperature-mortality association in 10 regions of England and Wales, 1993-2006. First-stage region-specific and pooled ( $95 \% \mathrm{Cl}$ as grey area) summaries at $22^{\circ} \mathrm{C}$ (left panel) and $0^{\circ} \mathrm{C}$ (right panel). Reference at $17^{\circ} \mathrm{C}$. 


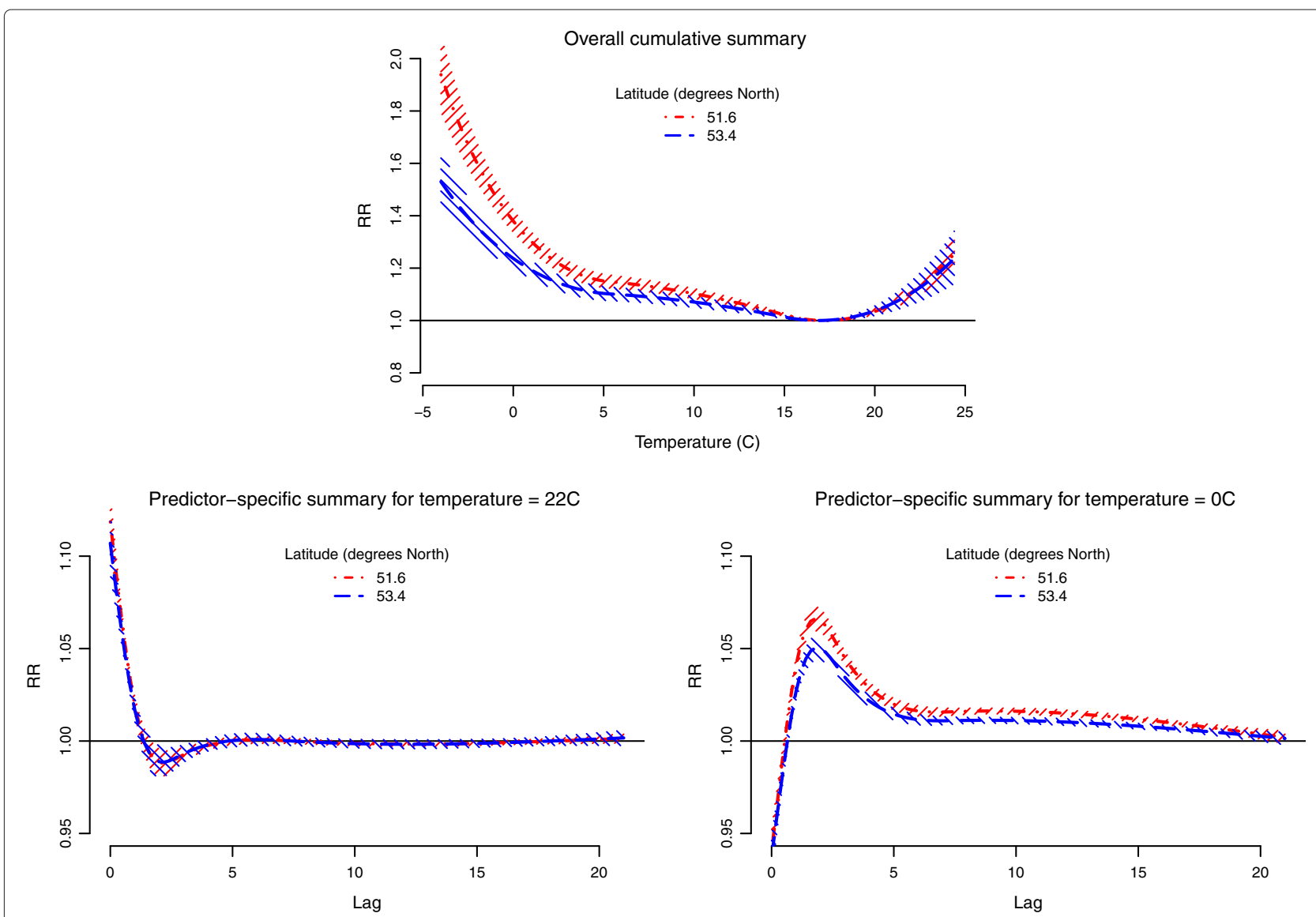

Figure 4 Pooled temperature-mortality association by latitude in 10 regions of England and Wales, 1993-2006. Predictions for the $25^{\text {th }}$ (dot-dashed line) and $75^{\text {th }}$ (dashed line) percentiles of latitude from meta-regression for overall cumulative summary (top panel), and predictor-specific summaries at $22^{\circ} \mathrm{C}$ (bottom-left panel) and $0^{\circ} \mathrm{C}$ (bottom-right panel). Reference at $17^{\circ} \mathrm{C}$. The $95 \% \mathrm{Cl}$ are reported as shaded areas.

to biases when the true underlying dependency is misspecified. The framework we propose, in contrast, require less assumptions or simplifications regarding the association in the first-stage model, but rather reduces the estimates to uni-dimensional summaries of a bi-dimensional fit. The advantages of this approach are exemplified by the comparison of the simpler alternatives with the bidimensionally flexible model, illustrated in the Results section. This methodology offers greater flexibility in the investigation of complex associations through a two-stage analysis.

Most of the limitations of DLNMs and multivariate meta-analysis of multi-parameter associations, previously discussed [5,12], identically apply to this framework. In particular, the issues of model selection and control for confounding pose important challenges, and are matters of current and future research. The issue of model selection is particularly relevant, due to the bi-dimensional nature of the models, where two independent bases are chosen to describe the dependency along predictor and lag spaces, respectively. In our example, we selected the bases a-priori for illustrative purposes, but model selection is clearly more problematic in applied analyses.

The problem of estimating perfectly correlated random components in the second-stage meta-analytical model, as described in the example, can bias upward the standard errors of the pooled estimates. This problem occurs in likelihood-based and method of moments estimation procedures of multivariate meta-analysis, as these estimators truncate the between-study correlations on the boundary of their parameter space $[16,19,23]$. Although in many cases this problem arises with small number of studies or when the amount of heterogeneity is negligible (and thus when a fixed-effects model is preferable), alternative approaches may be considered. First, different estimation methods can be applied, for example by imposing some structure to the between-study (co)variance matrix, or adopting a Bayesian approach that employ weakly informative priors models could avoid truncation of betweenstudy correlations. Also, alternative parameterization of the cross-basis functions may reduce the correlation pattern in the first stage and avoid estimation problems in the 
second-stage multivariate model. This issue needs to be explored further.

The definition of identical cross-basis functions in all the locations can be problematic in the presence of substantially different exposure ranges. In our example, the temperature distribution was similar across regions, and the placements of common knots was straightforward. However, this can be hardly generalized. The issue was previously discussed, and an alternative approach based on relative scale was proposed for pooling onedimensional functions [12]. The same method is applicable for bi-dimensional DLNMs. However, this limitation requires further research.

Estimation methods for DLNMs not requiring the completely parametric approach proposed here seems attractive and possible, in particular based on penalized likelihood [24] or Bayesian methods [25]. These estimation procedures also provide automatic selection methods. These options require the specification of a large number of parameters, which are then shrunk during the fitting procedures to reach a far smaller number of equivalent $d f$. However, the high dimensionality of the fitted model may present a problem for the second-stage multivariate meta-analysis, even when reduced to unidimensional summaries following (4)-(5). Techniques for meta-analysis of high-dimensional estimates are a topic of current and future research.

Potentially, the number of parameters of the secondstage multivariate meta-analysis can also be decreased by structuring the between-study (co)variance matrix of random effects. However, the extent to which such a choice can bias the estimates of fixed-effects parameters is not known. Moreover, this option is not yet available in the $R$ package mvmeta, and will be implemented and assessed in future analyses.

\section{Conclusions}

The extension of the DLNM framework presented here, involving the reduction of the complex two-dimensional fit to one-dimensional summaries, provides an improved method to study complex non-linear and delayed associations in two-stage analyses. Unlike previous approaches proposed so far, this method requires less simplification of the exposure-response shape or lag structure. This framework may be applied in any setting where non-linear and delayed relationships needs to be investigated in different populations or groups.

\section{Additional files}

Additional file 1: Online appendix. This pdf document provides additional information on the algebraic notation, on the software and $\mathrm{R}$ code, and on the time series data used in the example.
Additional file 2: Data. This csv file includes the time series data for the 10 regions of England and Wales during the period 1993-2006, used in the example.

Additional file 3: $\mathbf{R}$ scripts. This zip file contains $6 \mathrm{R}$ scripts. These are files with extension. $R$ which can be used to reproduce the results of the analysis in the example. They can be opened directly in $\mathrm{R}$ or read with a text editor.

\section{Abbreviations}

DLM: distributed lag model; DLNM: distributed lag non-linear model.

\section{Competing interests}

The authors declare that they have no competing interests.

\section{Author's contributions}

BA firstly conceived the idea of re-expressing summaries of DLNMs in terms of one-dimensional functions. AG then derived the algebraic expression. AG and BA contributed to the structure of the manuscript and the design of the analysis in the examples. AG implemented the methodology in the R software, performed the analysis, and took the lead in drafting the manuscript. BA contributed to drafting the manuscript. All authors read and approved the final version of the manuscript.

\section{Acknowledgements}

Antonio Gasparrini is currently funded by a Methodology Research Fellowship from Medical Research Council UK (grant ID G1002296). Ben Armstrong and Antonio Gasparrini were supported by a grant from Medical Research Council UK during the preliminary stage of the project (grant ID G0701030).

\section{Author details}

${ }^{1}$ Department of Medical Statistics, London School of Hygiene and Tropical Medicine, London, UK. ${ }^{2}$ Department of Social and Environmental Health Research, London School of Hygiene and Tropical Medicine, London, UK.

Received: 8 October 2012 Accepted: 17 December 2012 Published: 9 January 2013

\section{References}

1. Touloumi G, Atkinson R, Le Tertre A, Samoli E, Schwartz J, Schindler C, Vonk JM, Rossi G, Saez M, Rabszenko D: Analysis of health outcome time series data in epidemiological studies. EnvironMetrics 2004, 15(2):101-117.

2. Bell ML, Samet JM, Dominici F: Time-series studies of particulate matter. Annu Rev Public Health 2004, 25:247-280.

3. Dominici F, Samet JM, Zeger SL: Combining evidence on air pollution and daily mortality from the $\mathbf{2 0}$ largest US cities: a hierarchical modelling strategy. J R Stat Soc: Ser A 2000, 163(3):263-302.

4. Armstrong B: Models for the relationship between ambient temperature and daily mortality. Epidemiology 2006, 17(6):624-631.

5. Gasparrini A, Armstrong B, Kenward MG: Distributed lag non-linear models. Stat Med 2010, 29(21):2224-2234.

6. Dominici F, Daniels MJ, Zeger SL, Samet JM: Air pollution and mortality: estimating regional and national dose-response relationships. JAm Stat Assoc 2002, 97:100-111.

7. Samoli E, Touloumi G, Zanobetti A, Le Tertre A, Schindler C, Atkinson R, Vonk J, Rossi G, Saez M, Rabczenko D, et al.: Investigating the doseresponse relation between air pollution and total mortality in the APHEA-2 multicity project. Occup Environ Med 2003, 60(12):977-982

8. Baccini M, Biggeri A, Accetta G, Kosatsky T, Katsouyanni K, Analitis A Anderson HR, Bisanti L, D'Ippoliti D, Danova J, Forsberg B, Medina S, Paldy A, Rabczenko D, Schindler C, Michelozzi P: Heat effects on mortality in 15 European cities. Epidemiology 2008, 19(5):711-719.

9. Zanobetti A, Schwartz J, Samoli E, Gryparis A, Touloumi G, Atkinson R, Le Tertre A, Bobros J, Celko M, Goren A, Forsberg B, Michelozzi P, Rabczenko $D$, Aranguez Ruiz E, Katsouyanni K: The temporal pattern of mortality responses to air pollution: a multicity assessment of mortality displacement. Epidemiology 2002, 13:87-93.

10. Analitis A, Katsouyanni K, Biggeri A, Baccini M, Forsberg B, Bisanti L, Kirchmayer U, Ballester F, Cadum E, Goodman PG, Hojs A, Sunyer J, Tiittanen P, Michelozzi P: Effects of cold weather on mortality: results 
from 15 European cities within the PHEWE Project. Am J Epidemiol 2008, 168(12):1397.

11. Samoli E, Zanobetti A, Schwartz J, Atkinson R, Le Tertre A, Schindler C, Perez L, Cadum E, Pekkanen J, Paldy A, Touloumi G, Katsouyanni K: The temporal pattern of mortality responses to ambient ozone in the APHEA project. J Epidemio/ Community Health 2009, 63:960-966.

12. Gasparrini A, Armstrong B, Kenward MG: Multivariate meta-analysis for non-linear and other multi-parameter associations. Stat Med 2012 31(29):3821-3839.

13. Armstrong BG, Chalabi Z, Fenn B, Hajat S, Kovats S, Milojevic A, Wilkinson $P:$ The association of mortality with high temperatures in a temperate climate: England and Wales. J Epidemio/ Community Health 2011, 65(4):340-345.

14. Gasparrini A, Armstrong B, Kovats S, Wilkinson P: The effect of high temperatures on cause-specific mortality in England and Wales. Occup Environ Med 2012, 69:56-61.

15. Gasparrini A: Distributed lag linear and non-linear models in R: the package dlnm. J Stat Software 2011, 43(8):1-20.

16. Jackson D, Riley R, White IR: Multivariate meta-analysis: potential and promise. Stat Med 2011, 30(20):2481-2498.

17. Verbeke G, Molenberghs G: Linear mixed models for longitudinal data. New York: Springer-Verlag; 2000.

18. Nam IS, Mengersen $\mathrm{K}$, Garthwaite P: Multivariate meta-analysis. Stat Med 2003, 22(14):2309-2333.

19. Jackson D, White IR, Thompson SG: Extending DerSimonian and Laird's methodology to perform multivariate random effects meta-analyses. Stat Med 2010, 29(12):1282-1297.

20. Harville DA: Maximum likelihood approaches to variance component estimation and to related problems. J Am Stat Assoc 1977, 72(358):320-338.

21. van Houwelingen $H C$, Arends $L R$, Stijnen T: Advanced methods in meta-analysis: multivariate approach and meta-regression. Stat Med 2002, 21(4):589-624.

22. Anderson BG, Bell ML: Weather-related mortality: how heat, cold, and heat waves affect mortality in the United States. Epidemiology 2009, 20(2):205-213.

23. Riley RD, Abrams KR, Sutton AJ, Lambert PC, Thompson JR: Bivariate random-effects meta-analysis and the estimation of between-study correlation. BMC Med Res Methodology 2007, 7:3.

24. Zanobetti A, Wand MP, Schwartz J, Ryan LM: Generalized additive distributed lag models: quantifying mortality displacement. Biostatistics 2000, 1(3):279-292.

25. Welty LJ, Peng RD, Zeger SL, Dominici F: Bayesian distributed lag models: estimating effects of particulate matter air pollution on daily mortality. Biometrics 2008, 65:282-291.

Submit your next manuscript to BioMed Central and take full advantage of:

- Convenient online submission

- Thorough peer review

- No space constraints or color figure charges

- Immediate publication on acceptance

- Inclusion in PubMed, CAS, Scopus and Google Scholar

- Research which is freely available for redistribution

Submit your manuscript at www.biomedcentral.com/submit
() Biomed Central 